\title{
Influencia del fuego en el desequilibrio ecológico de la vegetación en la zona mediterránea de Chile: casos de estudio
}

\author{
Víctor Quintanilla Pérez \\ Departamento de Geografía. Universidad de Chile \\ Departamento de Ingeniería Geográfica. Universidad de Santiago de Chile \\ Email:vquintan@lauca.usach.cl
}

\begin{abstract}
RESUMEN
Se estudia los efectos del fuego sobre los cambios de vegetación nativa en Chile mediterráneo, entre aproximadamente $32^{\circ}$ y $33^{\circ} 40^{\prime}$ sur. Las formaciones predominantes corresponden al bosque esclerófilo y al matorral, lo cual en gran parte es el testimonio del bosque primitivo que hubo siglos atrás. El método geobotánico se apoyó previamente en el análisis temporal de fotos aéreas y en el trabajo de campo. Se hizo uso de parcelas de muestreo, líneas y censos para medir la composición, con recubrimiento antes y después de los incendios. En muchos sectores éstos se han producido desde hace más de 30 años. En las parcelas piloto, se han hecho hasta ahora mediciones y observaciones durante dos años. Se observa cambios en la estructura de las comunidades y la paulatina desaparición de algunas especies, pero en general predomina aún una recuperación de la mayoría de ellas. Se procura identificar cartográficamente las características de la vegetación con respecto al fuego y los cambios espaciales de su distribución. Hasta ahora se concluye que tres comunidades de plantas muestran diferentes estrategias para hacer frente al fuego: Lithrea - Cryptocaria con un período vegetativo largo y sostenido hacia la regeneración; Puya - Baccharis con un proceso más rápido aún y demostrando mayor resistencia; y las comunidades de palmas de Jubaea que en general no son dañadas. De modo preliminar se indican los efectos del fuego de estos últimos años, en bosques semihigrófitos andinos y en agrupaciones del sur de la zona mediterránea.
\end{abstract}

Palabras clave: Esclerófilo, fuego, Jubaea chilensis, Austrocedrus chilensis.

\section{Influence of forest fires on the ecological equilibrium of the mediterranean vegetation of Chile: study cases.}

\begin{abstract}
The effect of forestry wildfires on scrub and sclerophyllous forest in the mediterranean climate zone of Chile is studied, evaluating both the impact and the recovering capacity. The principal study was carried out in the coastal valley near Valparaíso and Viña del Mar cities. In the control area a phytogeographical study was carried out, with land survey and aerial photographic analysis, covering 30 years. The recovery of community structures was progresive, starting from the lower strata. Then was developed a more complex trend to revert towards a structure similar to the adult unburned stages. In the control area, a floristic study with intercepted line method was used. In the burnt area, the sequency of recovery size, covering (canopy) and volume of the principal species were studied during two consecutive years. After four or three years the sprouting, dead and new individuals were controled. Three communities with differents strategies of resistance to fire are detected: Lithrea-Cryptocaria, with vegetative sprout and long youthful phase, previous to ripenig; Puya-Baccharis with sprouting and ripenig, and community of Jubaea, which is not affected by the fire. The plant regeneration rate in shrub and herbaceous stratum was lower than expected and serious erosion processous were detected. Jubaea chilensis is in this moment in dangerous regeneration by the severe slope ravines. Some effects are shown, arising from forest fires in mountain humide woods and also in the southern side of the mediterranean zone.
\end{abstract}

Keys words: Sclerophyllous, fire, Jubaea chilensis, Austrocedrus chilensis. 


\section{INTRODUCCION}

Al igual que en otras zonas templadas del globo, el fuego ha sido un componente habitual de la evolución de los ecosistemas de gran parte del territorio chileno. A su vez la casi totalidad de los incendios producidos en el país son derivados de acciones humanas y en su mayoría corresponden a siniestros de tipo superficial. CARTRIWRIG (1966, en DONOSO 1990) estima que en la primera mitad del siglo $\mathrm{XX}$ se habrían quemado en el país alrededor de 1.200.000 hectáreas de bosques equivalentes a 4 mil millones de pulgadas de madera. Los fuegos recurrentes de verano se concentran principalmente en la zona mediterránea del país $y$, en no pocas veces, se han desplazado al sur de ella (por ejemplo entre los $40^{\circ}$ y $42^{\circ}$ sur). Los incendios forestales regulares se iniciaron desde alrededor de los años 60 aumentando casi anualmente, al punto que en el bienio de 1975 76 el número de siniestros por temporada supera los 2.000 en el centro y sur de Chile, para alcanzar ya en la temporada de 1994 - 95 a más de 5.500. Por otra parte, la superficie total quemada desde 1962 a esa fecha sobrepasaba las 100.000 hectáreas en el país.

En la Fig. 1 se muestra la evolución a nivel nacional del número de hectáreas quemadas y la relación entre la vegetación nativa y las plantaciones que se han incendiado en un período de tiempo de 20 años. Se observa por ejemplo las grandes extensiones quemadas en gran parte de la década de los años 80 , con respecto a la primera parte de la década de los $90, y$ el permanente incremento de la superficie quemada de vegetación autóctona. Durante la calurosa y seca temporada del fuego 1998 - 99 se desencadenaron grandes incendios en otros sectores de la zona mediterránea. En enero de 1999 se quemaron 14.000 hectáreas de pastizal, matorral y especialmente de bosques de Austrocedrus chilensis (ciprés de la cordillera) en la precordillera y montañas andinas de la VI Región, próximo a los 35 sur, al interior de San Fernando, principalmente en Sierras de Bellavista (Fig. 2). El incendio duró una semana y ardieron viejos cipreses de gran valor ecológico, puesto que esta conífera endémica de Sudamérica constituía en la región uno de los bosques de cordillera más longevos y mejor conservados del país.

En octubre del año 1998 (al sur de $\operatorname{los} 33^{\circ} \mathrm{S}$ ) el fuego consumió alrededor de 4.000 hectáreas de vegetación esclerófila y bosques de Nothofagus en los cerros del macizo de La

Figura 1

\section{Evolución del $\mathbf{N}^{\circ}$ de Hectáreas Quemadas Y relación entre Vegetación Nativa y Plantaciones en Chile Temporadas 1975 - 1995}

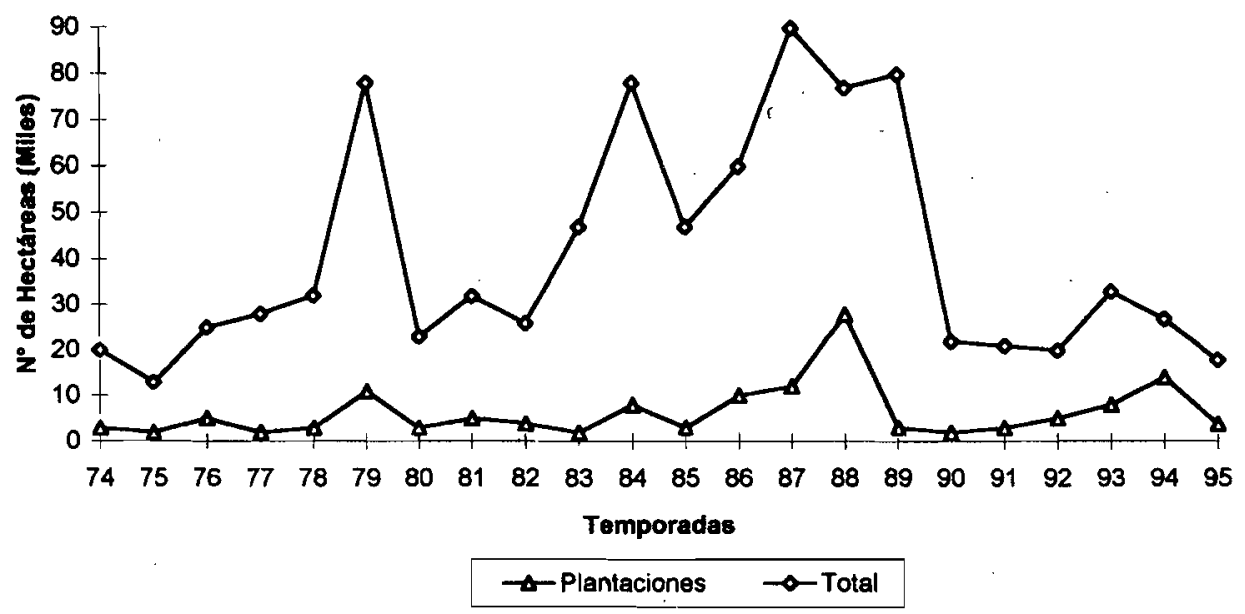

Fig. 1. Evolution of bourned area between 1975 and 1995. 


\section{Zona Mediterránea Típica de Chile}
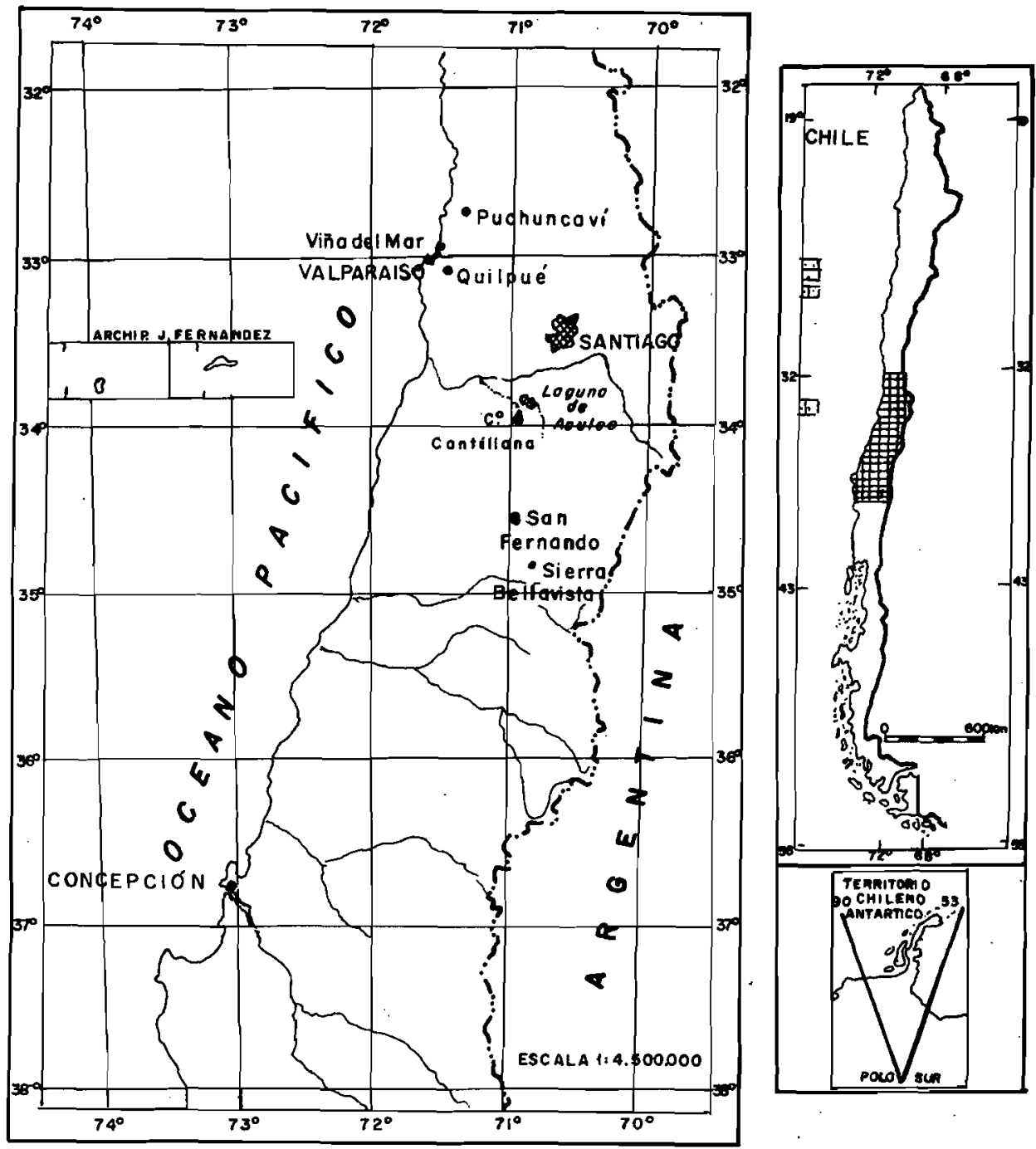

Fig. 2. Locational map.

Cantillana frente a la Laguna de Aculeo (Fig. 2). En estos cerros se conservaba uno de los bosques esclerófilos en mejor estado de equilibrio que existen en el interior de la Región Metropolitana. Lo mismo se puede indicar para los bosques de Nothofagus, los cuales estaban acompañados de un rico cortejo florístico de carácter higrófito.

En la segunda semana de febrero 1999 se desencadenaron numerosos fuegos en la depresión central y en la cordillera costera en- tre $37^{\circ}$ y $38^{\circ} \mathrm{S}$, arrasando 18.000 hectáreas vegetales conformadas por 12.000 hectáreas de plantaciones de pino insigne y eucaliptos y 6.000 hectáreas de matorral y bosque nativo. Por otra parte los daños alcanzaron a viviendas e infraestructuras agrícolas de comunas rurales como Bulnes, Quirihue, Hualqui, Chiguayante y Mulchén y sectores próximos a la ruta de Concepción a Bulnes. Durante una semana se sucedieron 8 focos de incendios casi al mismo tiempo, transformándose algunos de ellos en una cortina de fuego y humo de $3 \mathrm{kms}$. de ancho 
Figura 3

\section{Localización de los InCENDIOS Forestales en la VIII Región de Chile, ocurridos a fines de Febrero de 1999.}

En total se quemaron 18.000 has. de vegetación

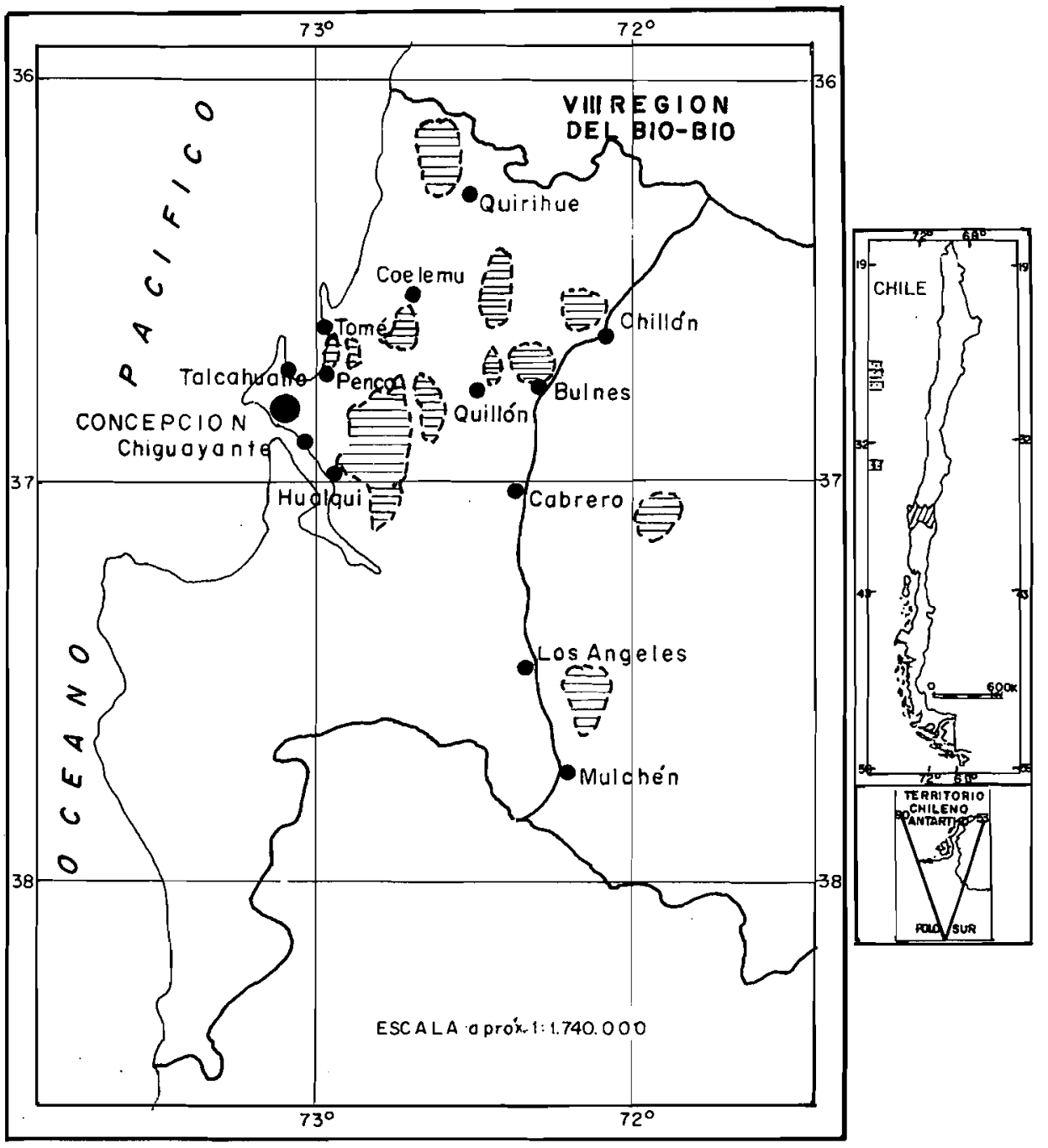

Fig. 3. Location of fires produced during february of 1999 in the Concepción region.

por 25 de largo (Fig. 3). Posteriormente, el 6 de Mayo de 1999 en la provincia de Valparaíso (alrededor de $\operatorname{los} 33^{\circ} \mathrm{S}$ ), se produjo uno de los incendios más intensos de su historia, el cual duró tres días. Con el fuerte viento, a causa de la alta temperatura del día, ardieron 80 hectáreas de eucaliptos y 20 de matorral. En marzo de 2000 se quemaron más de 600 hectáreas de pastizal, matorral y bosque nativo, durante cuatro días en el interior de la comuna de Puchuncaví al norte de Viña del Mar.

Debe señalarse que actualmente en Chile las plantaciones artificiales (Pinus radiata, Eucaliptus globolus, Populus spp.) alcanzan a 1.500.000 hectáreas y continúan en aumento. La región con mayor superficie de plantaciones corresponde a la VIII, localizada entre los $37^{\circ}$ 
y $38^{\circ} \mathrm{S}$, con un $39 \%$ del total nacional y seguida de la IX Región, situada entre los $38^{\circ}$ y $39^{\circ} \mathrm{S}$, con un $24 \%$. Por otra parte, la proporción conjunta de pastizales y de matorrales quemados es muy superior a la de los bosques artificiales, a excepción de lo que ardió al sur de los $38^{\circ}$. De las unidades de vegetación que arden durante la temporada del fuego al Norte de los $34^{\circ}$ (V Región), los bosques y el matorral esclerófilo adquieren un porcentaje significativo en comparación a los bajos valores que alcanzan los fuegos en los repoblamientos artificiales (Fig. 4). La causa de estos fuegos es exclusivamente antrópica. Tránsito, recreación, quema de desperdicios agrícolas, juegos de niños e incendiarios son los responsables de la casi totalidad de los siniestros en Chile. También debido a este impacto, la cubierta vegetal nativa de la zona mediterránea va hacia sucesivos y rápidos cambios de composición y estructura como consecuencia de la alta frecuencia de incendios iniciados regularmente desde casi cuatro décadas.

El fenómeno de los incendios forestales tiene una clara manifestación territorial (CHUVIECO et al.1997 - 98). El propósito de este trabajo es entregar un avance de estudios sobre modificaciones en formaciones vegetales nativas, a causa del efecto recurrente de fuegos de verano.

\section{MATERIALES Y MÉTODOS}

Aunque la región de interés es toda la zona

FIGURA 4

$$
\begin{array}{r}
\text { V Región. } \\
\text { Distribución de los tipos de } \\
\text { combustible quemados, } \\
\text { temporadas } 1990-1995
\end{array}
$$

$$
\begin{array}{r}
\text { Promedio Temporadas } \\
1990-1995
\end{array}
$$ Tipo de Combustible Forestal \\ Promedio Temporadas}

mediterránea del país, las investigaciones se concentran en dos tipos de sectores con comunidades nativas: donde hubo en las últimas tres décadas incendios recurrentes y donde el tamaño de la superficie incendiada afectó a grandes áreas fitogeográficamente importantes. Dentro del primer caso está el área del cordón costero de la denominada V Región (32 a $33^{\circ}$ aproximadamente (Fig. 5). En el segundo caso se encuentran las áreas cordilleranas de la VI Región (Sierras de Bellavista) y sectores costeros de la VIII Región.

A fines de Febrero de 1996 y principios de Enero de 1997 se produjeron sendos incendios en quebradas y laderas periféricas a las ciudades de Viña del Mar y Quilpué. En estas colinas, de alturas medias de $220 \mathrm{mts}$., se quemaron 700 hectáreas en 1996 y alrededor de 1.600 en 1997. Dicha área se quemaba por séptima vez en los últimos 14 años. 510 hectáreas correspondían a plantaciones de pino insigne y eucaliptos $\mathrm{y}$ el resto a matorral esclerófilo, palmas chilenas (Jubaea chilensis) y pastizales. Según censos de CODEFF (Corporación Defensa Flora y Fauna), en estas quebradas existirian poco más de 3.000 palmas. La vegetación quedó destruida aproximadamente en un $70 \%$ y además el fuego amenazó a poblaciones de habitantes de la parte alta de Viña del Mar y Quilpué.

Se trabajó con la hipótesis de una modificación sustantiva del tapiz vegetal primitivo de estos sectores, por efecto de incendios sucesivos. Este efecto se ha confirmado en otros ecosistemas

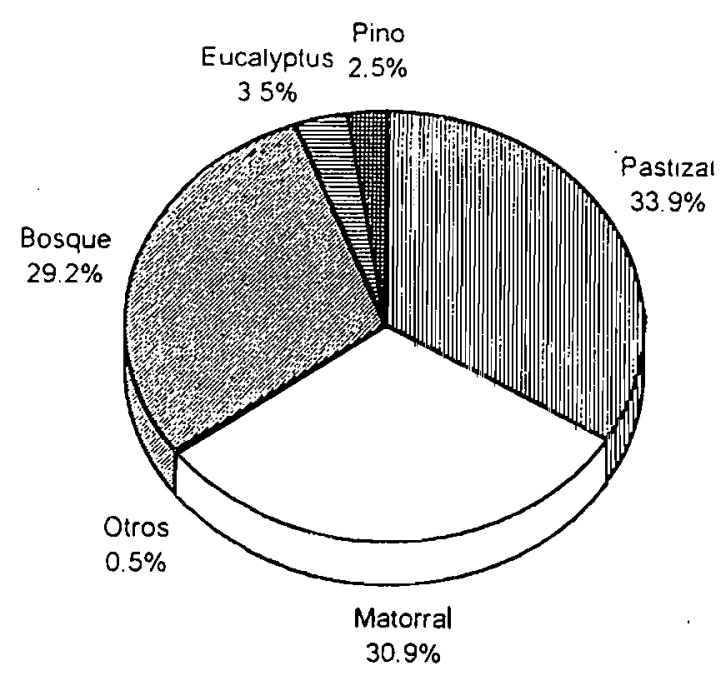

Fig. 4. Distribution of types of bourned plants or communities in the Valparaíso region (during 1990 - 1995). 
mediterráneos, donde hace tiempo se hace un seguimiento a las comunidades incendiadas .(PAPIÓ y TRABAUD 1999).

La evolución de estos cambios se representará principalmente a través del método de cartografía diacrónica propuesto por TRABAUD ( 1981) y en QUINTANILLA (1998 y 1999). Se efectuó primero un análisis para identificar, a través de bibliografía y descripciones históricas, la vegetación que existía al menos durante el siglo pasado en las colinas costeras de la V Región. La búsqueda de cartas vegetales y de usos del suelo fue poco provechosa. El análisis en escalas medias de fotos aéreas de los años 1962, 1983 y 1994 permitió obtener una visión global respecto a la distribución espacial de las unidades de vegetación presentes en la actualidad. Esta interpretación permitió efectuar una primera selección de las áreas piloto a ubicar en el trabajo de campo. A continuación se determinaron en terreno las interacciones de los principales factores ecológicos de las áreas de estudio, entes de llevar a cabo los muestreos - Se seleccionaron 12 parcelas de $60 \times 40$ metros, próximas entre ellas, pero considerando variables diversas: sectores no quemados, sectores con quema superficial y sectores de incendios de copa. En todas las parcelas se elaboraron muestreos fitosociológicos sobre todo de árboles y arbustos, aplicando el método de punto interceptado (BRAUN-BLANQUET 1951), para tener una referencia cuantitativa de la vegetación, previa y posterior a los incendios. Estas mediciones se hicieron

\section{Figura 5}

\section{ÁREA DE INCENDIOS RECURRENTES}

\section{EN LA V Región en los ÚLTIMOS 15 AÑos}

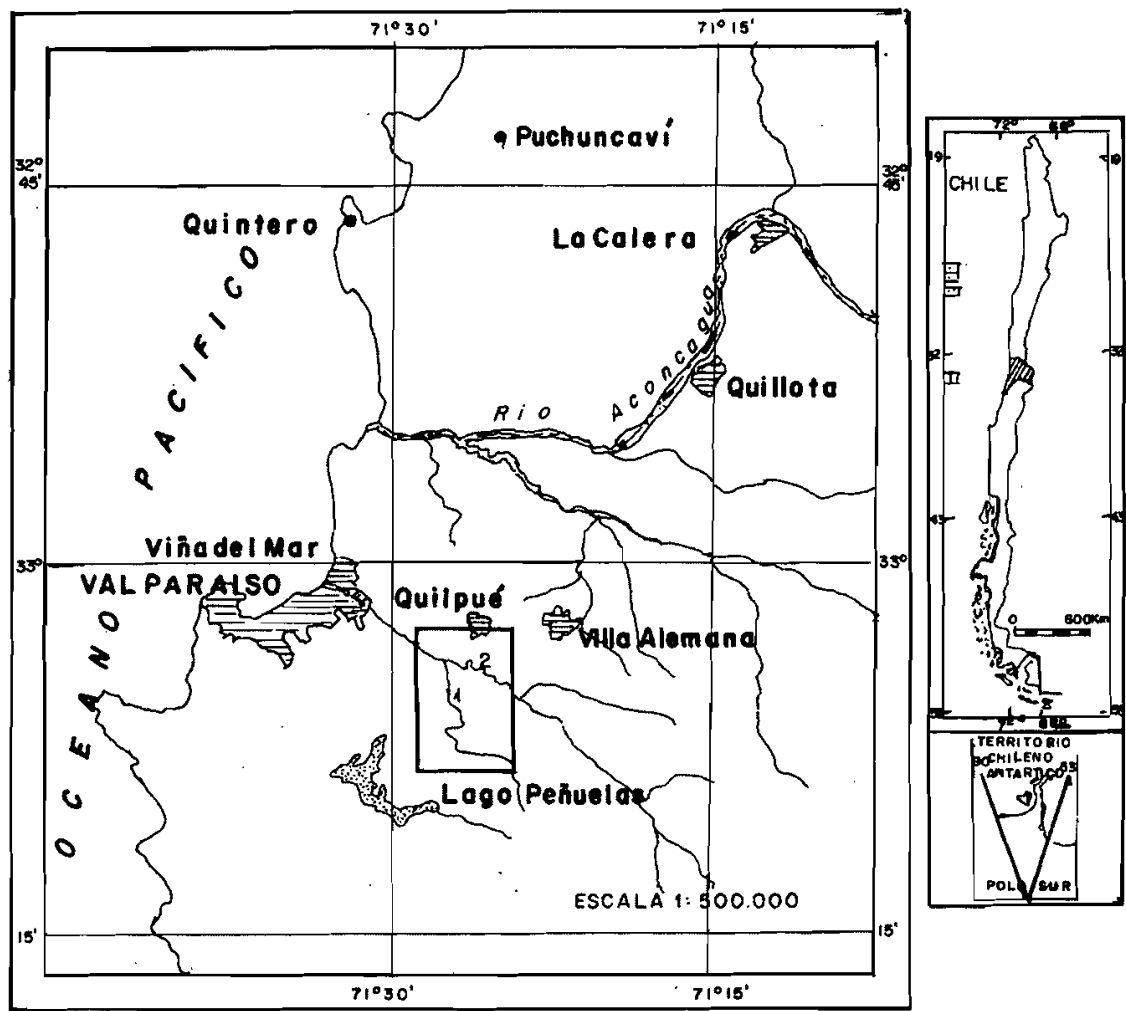

1.- Quebrada Siete Hermanos

2.- Quebrada El Quiteño

Fig. 5. Location of fires in the Valparaíso region during the last 15 years. 
aproximadamente cada 40 días durante un tiempo de dos años. Se va calculando la altura y volumen inicial de los individuos (la talla que tenían antes de ser quemados), considerando la altura de los palos calcinados y el diámetro probable de cobertura de copa. También se está estudiando la secuencia de la relación, entre especies preexistentes y nuevas, en la ocupación de los espacios libres.

\section{RESULTADOS}

De 21 censos fitosociológicos en parcelas de $40 \mathrm{~m} 2$ en las colinas costeras de la $\mathrm{V}$ Región, se destaca el valor de presencia de las especies en las parcelas censadas (Tabla 1).

Al cabo de dos años de producidos los últimos incendios y entre un año y el siguiente en que

\section{TABLA 1}

\section{Valores de Presencia de Plantas más constantes en Muestras de Terreno (Parcela de 40M²) IX - 1996 a VI - 1997.}

\begin{tabular}{|c|c|c|c|c|c|c|c|c|c|c|c|c|c|c|c|c|c|c|c|c|c|c|}
\hline $\mathrm{N}^{\circ}$ de Censo & 1 & 2 & 3 & 4 & 5 & 6 & 7 & 8 & 9 & 10 & 11 & 12 & 13 & 14 & 15 & 16 & 17 & 18 & 19 & 20 & 21 & 22 \\
\hline Altura (m) & 230 & 230 & 240 & 250 & 250 & 250 & 260 & 260 & 280 & 280 & 300 & 300 & 3503 & $350=$ & 390 & 390 & 400 & 410 & 410 & 410 & 420 & 420 \\
\hline Exposición & $\mathrm{W}$ & $\mathrm{E}$ & $w$ & $\mathrm{E}$ & $\mathrm{W}$ & $\mathrm{E}$ & W & $\mathrm{E}$ & SW & $\mathrm{E}$ & SW & $E$ & $\dot{\mathrm{S} W}$ & E & SW & $E$ & SW & $\mathrm{E}$ & SW & $\mathrm{E}$ & SW & $\mathrm{E}$ \\
\hline Pendiente (\%) & 5 & 5 & 10 & 10 & 20 & 10 & 15 & 15 & 20 & 20 & 20 & 20 & 15 & 15 & 10 & 10 & 20 & 20 & 25 & 25 & 15 & 15 \\
\hline \multicolumn{23}{|l|}{ Estrato Arbóreo } \\
\hline Lithrea caustica & 4 & 2 & 1 & 1 & 18 & 3 & 13 & 3 & 1 & 2 & 7 & 8 & 2 & 1 & 4 & 1 & 2 & 1 & 2 & 2 & & 3 \\
\hline Cryptocaria alba & & & & & 1 & 2 & 1 & 1 & 3 & 1 & 1 & & 1 & & 2 & . & & & & & & 1 \\
\hline Peumus boldus & 1 & & & 10 & 12 & & & 1 & 2 & & & 2 & 2 & & 2 & . & 1 & 1 & 2 & & 2 &. \\
\hline Quillaja saponaria & & 1 & & 1 & 2 & . & 2 & 1 & 7 & & 1 & 1. & & 2 & 1 & 1 & 1 & 2 & & 2 & & 2 \\
\hline Schinus polygamus & & & & 4 & 1 & & & & & 1 & 2 & 1 & & & 1 & 1 & & 1 & . & 1 & & 1 \\
\hline Jubaea chilensis & & & & & & & & 1 & 2 & 1 & 2 & & & & & . & & & &. & &. \\
\hline Drymis winteri & . & $\cdot$ & & & . &. & & 7 & & &. & & & &. & . & & 2 & 7 & 1 & 2 & 1 \\
\hline \multicolumn{23}{|c|}{ Estrato Arbustivo y Herbáceo } \\
\hline Adenopeltis colliguaja & & & & 2 & & 1 & & & 4 & & 2 & & & 3 & . & 2 & & 1 & & & & \\
\hline Baccharis concava & & & & & &. & & 7 & 9 & 12 & 4 & & & 1 & 13 & 9 & 12 & 16 & & & & . \\
\hline Baccharis tosmarinifolia & & 5 & & 11 & & 17 & 3 & 8 & & & 6 & & & 3 & & 6 & & 7 & & 5 & 8 & 4 \\
\hline Podantus mitiqui & & & & & 9 & 4 & & 5 & & 6 & & 2 & & 7 & & 8 & & 3 & . & 4 & & 7 \\
\hline Cassia stipulacea & . & . & 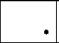 & 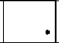 & . &. & 7 &. & 2 & . & 4 & 16 & & 3 & . & 22 & . & . & 19 & 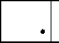 & & 3 \\
\hline Trevoa trinervis & . & . & 21 & 7 & & 12 & & & 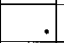 & & . & 7 & &. & . & 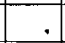 & & . &. & . & & . \\
\hline Echinopsis chilensis & . & 1 &. & & . & . & 3 & . & 1 & . & 1 & . & & . & 1 & . & . &. &. & . & & . \\
\hline Puya chilensis & & & 7 & & . &. &. & 3 &. & 1 & . & 4 & & . & . & 3 &. & 2 &. & & & . \\
\hline Escallonia pulverulenta & & 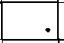 & 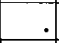 & & . &. & . &. & . & 1 &. & & 7 &. & 4 & . & 2 & 2 & 3 & & 1 & . \\
\hline Eringium paniculatum & 6 & 2 & 3 & 2 & 7 & 5 & 3 & 6 & 15 & 4 & 21 & 3 & 11 & 6 & 16 & 11 & 20 & 5 & 18 & & 23 & 4 \\
\hline Rubus ulmifolius & 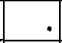 & $\cdot$ &. & &. &. &. &. & 3 &. & 6 & 1 & 5 & . & 7 &. & 3 & . &. & & & . \\
\hline Chusquea cumingii & . &. &. & & . & . & . & . & . &. &. & 7 & . &. & 6 & . & 6 & . & . & & . & . \\
\hline Colliguaja adorifera & & &. & & 7 & 9 & 6 & 11 & 10 & 12 & 11 & 9 & 3 & 7 & 6 & 4 & . & 5 & 2 & & 7 & 7 \\
\hline Gallium aparine & 11 & 10 & 6 & 21 & 5 & 15 & 12 & 2 & 27 & 9 & 23 & 6 & 14 & 22 & 35 & 15 & 13 & 11 & 12 & 19 & 22 & 19 \\
\hline Aristotelia chilensis & & & $\cdot$ & & & &. & & 4 & . & & & &. & 1 &. & 4 & 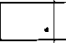 & 3 & & & \\
\hline Ribes punctatum & & & 7 & & 10 & 6 &. & & 5 & 9 & & 12 & & 7 &. & 4 & 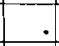 & 4 &. & & & \\
\hline Muehlenbeckia hastulata & 7 & 9 & 3 & 5 & 8 &. & 12 &. & 16 &. & . & 10 & 7 & 6 & 3 & 6 &. &. &. & &. & . \\
\hline Lobelia salicifolia & &. & $\cdot$ & 4 & & 5 & 1 & 6 & 2 & 7 & 2 & 3 & ] & 5 & 2 & 3 & & 4 & . & 3 & 1 & 2 \\
\hline Colletia spinossa & . &. &. & & &. & . &. & & & & . &. & . & . & . & & &. & 3 & 2 & 1 \\
\hline Pasithaea coerulea & & 4 & & & & 7 & 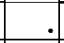 & 7 & & & &. & . & . &. & . & & 4 & 2 & 1 & 2 & 1 \\
\hline Satureja gilliessii & 4 & 7 & 3 & 4 & 17 & 2 & 14 & 11 & 21 & 9 & 3 & 6 & 12 & 3 & 13 & 6 & 2 & 6 & 1 & 5 & 3 & 2 \\
\hline Calceolaria thyrsifolia & & & 4 & & 9 & 7 & 1 & 6 & 5 & 8 & 3 &. & . & 4 & 7 & . & 11 &. & 12 & & 10 & 2 \\
\hline Cestrum parqui & & & 1 & 1 & 3 & 2 & 6 & 3 & 1 & 3 & 7 & 9 & 5 & 4 & 2 & 1 & 1 & 1 & 3 & 2 & 4 & 2 \\
\hline Gnaphalium sp. & & & 1 & 4 & 2 & 5 & 2 & $\therefore$ & 3 & & 4 & & 5 & 6 &. & 3 & & 3 & & 4 & & \\
\hline Crysanthemun parthenium & & & & 13 & 10 & 8 & 14 & 12 & 7 & 12 & 17 & & 5 & 3 & 2 & 9 & 8 & 5 & 3 & 6 & 11 & 10 \\
\hline Puya venusta & & $\cdot$ & & & & & & 1 & & & & & &. &. & 3 &. &. &. & 1 & & \\
\hline
\end{tabular}


se sucedieron nuevos fuegos (1996 - 97 y 1997

- 98) se anotaron los individuos rebrotados, no rebrotados (muertos) y nuevos de cada especie encontrada, para posteriormente calcular el porcentaje de rebrote, mortalidad y capacidad de colonización. Se constató que el efecto inmediato de los fuegos, fue la combustión total de los estratos herbáceo y arbustivo y más o menos un $60 \%$ del arbóreo. Los suelos de las quebradas con una pendiente superior al $10 \%$ denotaron la evidencia de procesos erosivos.

Evolutivamente, se observa que el fuego ha operado como un organismo de selección sobre las plantas. Por ejemplo, ha ocurrido rebrote de yemas protegidas por la corteza en el caso del eucalipto, incremento de la floración entre las plantas resistentes al fuego en el caso de Lithrea caustica, o retención de semillas en la planta a la espera de condiciones favorables. El alto desarrollo herbáceo en las comunidades vegetales recién quemadas ès atribuible tanto a la liberación de semillas retenidas por las plantas, como a la germinación de las semillas transportadas desde fuentes vecinas o bien que permanecían en reposo en el suelo (BALDUZZI et al. 1980 - 81).

Se advierte la pérdida de la cubierta vegetal del sustrato, lo cual permite que los suelos estén más susceptibles de ser erosionados por la acción de la lluvia y del viento, elementos que arrastran los subhorizontes superficiales del suelo. Esto implica también la pérdida de las semillas, lo cual se traduce en el retardo de la eventual regeneración de las plantas. Considerando esto último, se observa que la composición florística de la comunidad puede variar, siendo éste un indicador importante de modificaciones en el ecosistema. No obstante y de acuerdo con AVILA et al. (1988), se comprueba que en ésta área existen especies denominadas pioneras como Muehlenbeckia hastulata, Baccharis rosmarinifolia $\mathrm{y}$ Eringium paniculatum, las que tienden a colonizar espacios abiertos dejados por el paso del fuego. En las laderas de quebradas en exposición al mar se observó que esta regeneración y colonización es más activa.

Se aprecia que, después de un incendio, gran parte de la vegetación quemada tiende a regenerarse al poco tiempo aunque no se restablezca la misma estructura y fisonomía del paisaje preexistente antes del fuego. Gracias a las propiedades morfológicas y fisiológicas de las plantas, apoyadas por un mínimum de humedad, ellas pueden volver a restablecerse. Así, una de las formas de recuperación es a través de la germinación de semillas que nacen en el mismo lugar quemado o bien logran colonizar esos espacios a través del transporte por el viento, aves o mamíferos. Esto puede ocurrir en todas las formas, desde plantas anuales hasta árboles. Otro medio de recuperación de la cubierta vegetal es a través del desarrollo de yemas (puntas de posible rebrote) que no han sido afectadas por el fuego. La recuperación de yemas puede darse porque se encuentran bajo el suelo en las hierbas perennes y algunos arbustos, o bien se hallan protegidas del fuego por la corteza del árbol o arbusto. El rebrote experimentado en árboles y arbustos produce una rápida recuperación del follaje perdido, con renovación de los órganos de la planta y variaciones en su forma original. Cuando los individuos del estrato arbustivo desarrollan brotes a partir de yemas subterráneas se generan renovales, reproduciéndose a través de una multiplicidad de tallos que emergen simultáneamente del suelo (BALDUZZI et al. 1980 - 1981).

A poco tiempo de producidos los incendios, comienza el rebrote de algunas plantas activadas principalmente por la neblina costera. A los 21 días de desencadenados los fuegos, se detecta el rebrote de Eringium fasciculatum, arbusto perenne de 60 a $80 \mathrm{cms}$. de altura. Entre los árboles, Lithrea caustica rebrota a su vez a partir del lignotuber en la producción de ramas nuevas que nacen de un mismo punto y se inicia su regeneración más o menos a los 45 días de generado el incendio. Otro árbol esclerófilo con una relativa regeneración rápida en el lugar ha sido Cryptocaria alba, aunque su rebrote se ve frenado a causa de la fuerte herbivoría sobre los renuevos de parte de los roedores y el ganado vacuno. Según VILLASEÑOR y SAIZ ( 1993) su crecimiento se acelera sólo después de la primavera, aunque de modo menos rápido que en el litre. Algo similar sucede con otro abundante árbol del bosque esclerófilo como es Quillaja saponaria.

Los arbustos Muehlenbeckia hastulata (arbusto semitrepador), Podanthus mitiqui y Baccharis rosmarinifolia demostraron una mayor capacidad de recuperación y colonización estando casi todos desarrollándose en la temporada del fuego del año siguiente. No ocurre lo mismo con otros arbustos del matorral 
y que demuestran una regeneración más lenta. Así, Trevoa trinervis, Colliguaja odorifera, Baccharis paniculata, Adesmia phylloidea y Adesmia arborea, que se desarrollan en la primera estación de crecimiento en los espacios antes quemados, lo cual podría atribuirse en parte al aumento de la intensidad lumínica a que quedan expuestas las ramas.

En lo que respecta a la cubierta herbácea del matorral, ésta se recupera rápidamente después de las primeras lluvias. Sin embargo, al cabo de estos años de observaciones se denotan cambios importantes particularmente en el tamaño y número de individuos por unidad de superficie (densidad). Al parecer hay especies nativas que tienden a disminuir su densidad, en cambio otras preferentemente introducidas, tales como Avena barbata, Erodium cicutareum, Urtica urens y Carduns pynocephalus (malezas cosmopolitas) tienden a aumentar significativamente.

Esta continua pérdida y debilitamiento de la cubierta vegetal ha producido, en numerosos sectores de pendientes superiores al $10 \%$, procesos erosivos por acción de las precipitaciones y del viento. Este fenómeno implica además la pérdida de las semillas de ese suelo lo que se refleja en el retardo de la eventual regeneración del ecosistema. Por tanto hay variación de la composición florística de las comunidades.

No obstante aquí también se comprobaron fenómenos que habían constatado autores an-

FIGURA 6

\section{Extracto de la Carta de Formacines Vegetales Combustibles de los Cordones Montañosos Costeros de Chile Central Quintanilla 1998}

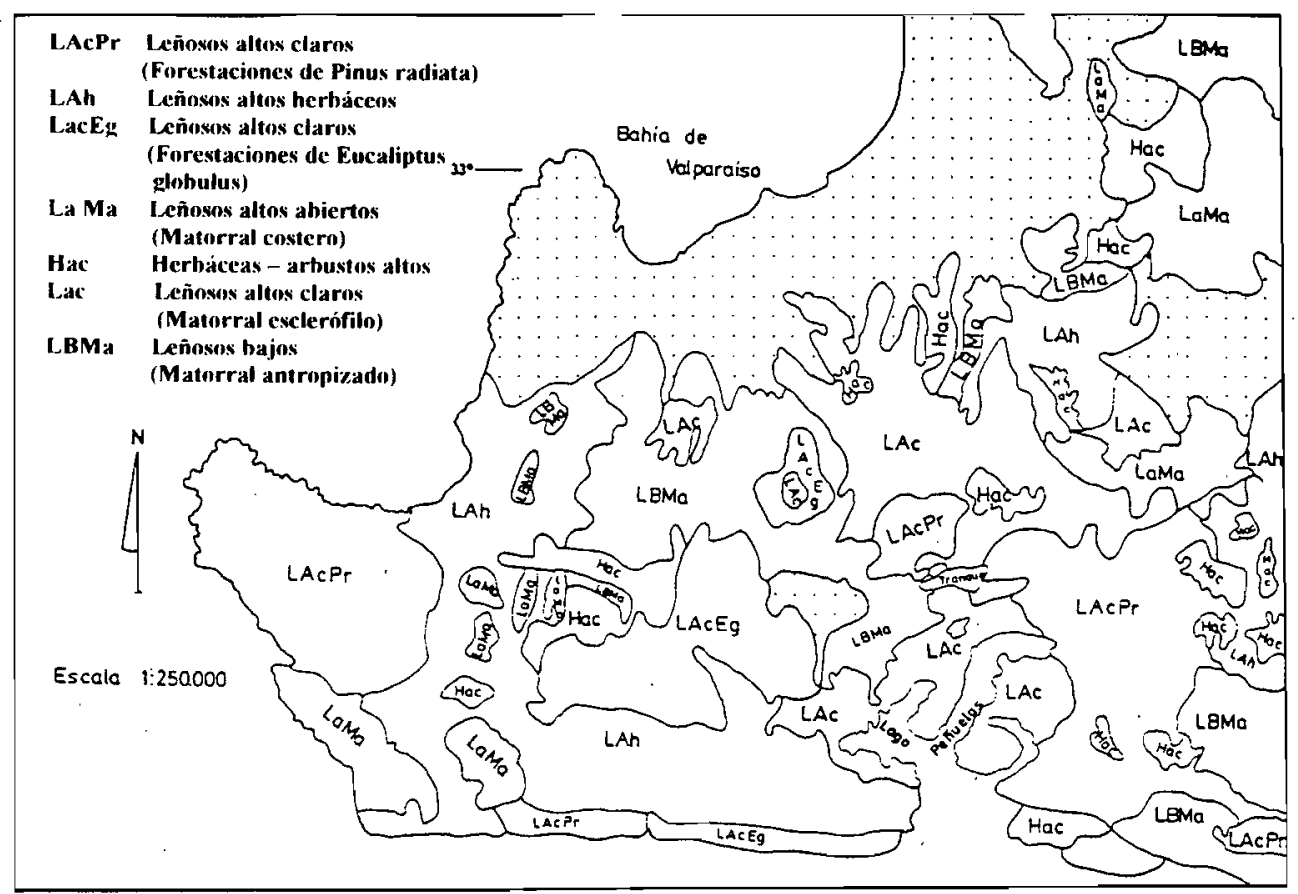

Fig. 6. Map of plant formations in the Valparaíso region. 
FIGURA 7

Perfil tipo de Vegetación Mediterránea Arbórea y Arbustiva antes y después del Fuego
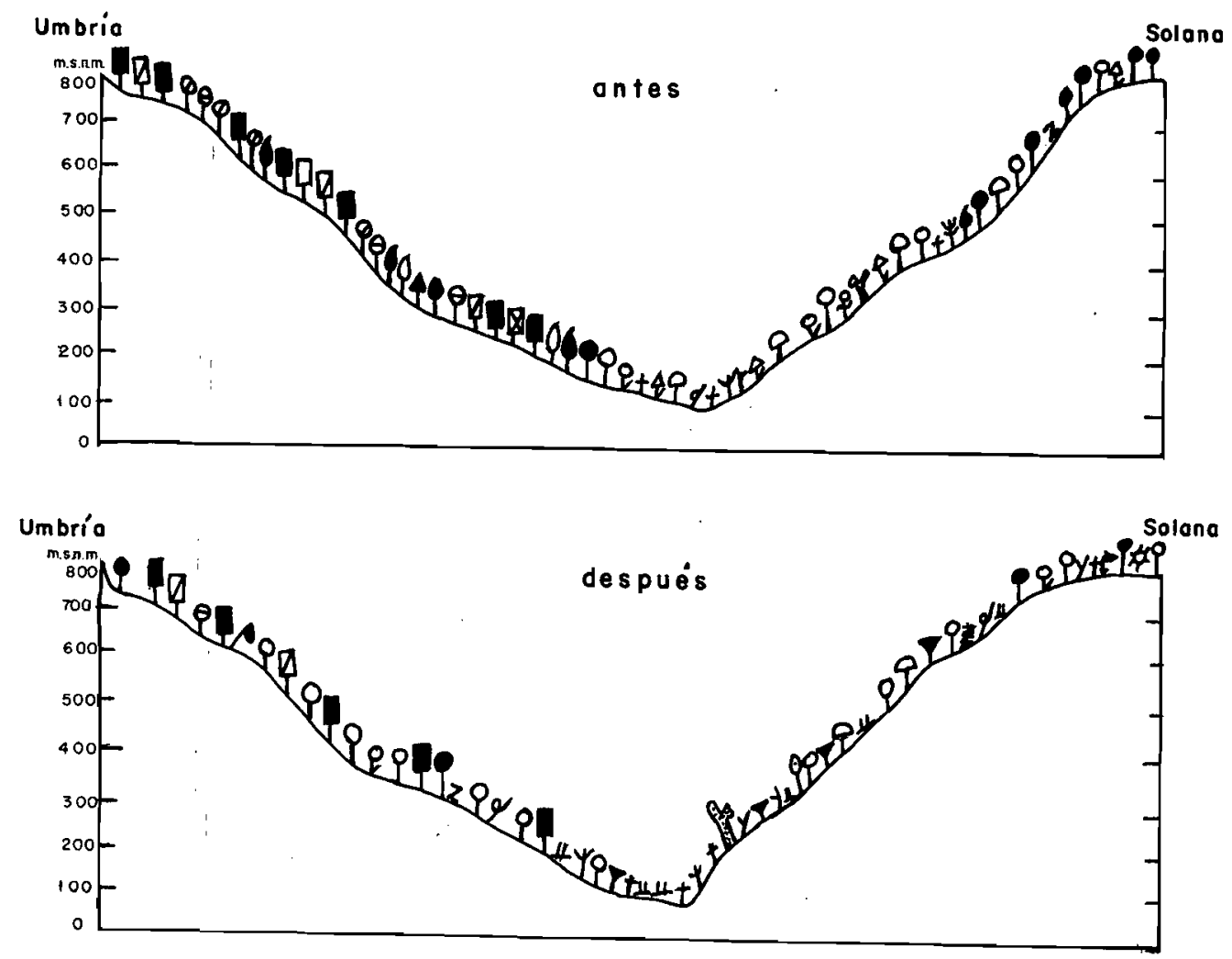

\section{LEYENDA}

$\begin{array}{ll}9 & \text { Acacia caven } \\ 4 & \text { Colliguaja adorifera } \\ q & \text { Podanthus mitiqui } \\ Y & \text { Trevoa trinervis } \\ + & \text { Bucchoris Ilnearis } \\ \rightarrow & \text { Fluoreensia thurifera } \\ 4 & \text { Muehlenbecckia hastulata } \\ 9 & \text { Cesirum Palqui } \\ 9 & \text { Lithrea caustica } \\ q & \text { Trichocereus chilensis } \\ \downarrow & \text { Puya chilensis } \\ \uparrow & \text { Quillaja saponaria } \\ \theta & \text { Margyricarpus pinnatus } \\ 7 & \text { Sphacele saviae } \\ Q & \text { Lobelia saalicifolia } \\ z & \text { Baccharis concava }\end{array}$

\begin{tabular}{|c|c|}
\hline 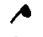 & Escallonia pulverulenta \\
\hline$q$ & Adesmia arborea \\
\hline & Schinus latifolius \\
\hline & Cryptocaria alba \\
\hline 8 & Peumus boidus \\
\hline 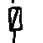 & Beilschmiedia miersii \\
\hline 9 & Azara celastrina \\
\hline$\varphi$ & Drymis winteru \\
\hline$\Delta$ & Escallonia illinita \\
\hline$\varphi$ & Aristotelia chilensis \\
\hline \$ & Eupatorium glechonophyllum \\
\hline$\theta$ & Myrceugenia exsucca \\
\hline$\sqrt{2}$ & Chusquea cumingii \\
\hline & Rubus ulmifolius \\
\hline 2 & Conium maculatum \\
\hline
\end{tabular}

Fig. 7. Typical profile of plants in opposite slopes, before and after a fire. 
tes citados (VILLASEÑOR y SAIZ 1992-1993) en fuegos producidos en otros sectores de la $\mathrm{V}$ Región. Surgen especies pioneras como el "quilo" (Muehelenbeckia hastulata) la "chilca" (Baccharis sp.) y el "corontillo" (Eringium paniculatum) que tienden a colonizar los espacios abiertos dejados por el fuego. Otra respuesta de los arbustos recuperados después del fuego, es la gran cantidad de flores que se se encontró en gran número de plántulas, las cuales no son tan comunes en las comunidades maduras del matorral.

\section{DISCUSION}

Los procesos meteorológicos asociados con los índices de riesgo de incendios en zonas mediterráneas (MILLAN et al. 1998 y JULIO 1993), han sido establecidos en diversos trabajos. Otros elementos que actúan en la zona, junto a los factores meteorológicos, son las características de la vegetación. Los árboles en su gran mayoría son esclerófilos, los arbustos en general son deciduos de verano y las hierbas son terófitas o geófitas con descanso estival. (ARAYA y AVILA 1981).

El bosque esclerófilo húmedo y mesotérmico era típico de la zona, predominando comunidades de Cryptocaria alba (peumo), Peumus boldus (boldo), Beilschmiedia miersii (belloto) y Quillaja saponaria (quillay). En los fondos de quebradas y sobre laderas de suaves pendientes o en fondos de valle, eran más constantes los bosques de Quillaja saponaria, Schinus latifolius (molle) y Lithrea caustica (litre). Dada la estructura continua que en un principio tuvieron estos bosques, resultaron ser con el tiempo las agrupaciones más afectadas por el fuego (QUINTANILLA 1983). En los sectores de alta humedad freática y próximos a cursos de agua, se desarrollaban bosques de tipo higrófito con presencia especialmente de Crinodendron patagua (patagua), Myrceugenia exsucca (arrayán) y Aristotelia chilensis (maqui). En lo que respecta a la vegetación arbustiva destacan los matorrales xéricos de laderas de solana y con predominio de comunidades de Colliguaja odorifera (colliguay), Trevoa trinervis (tebo), Fluourensia thuriphera (maravilla del campo) junto a las comunidades xéricas de Puya chilensis o $P$. berteroana (chagual). Esta última bromeliácea genera controvertidas versiones sobre un posible carácter, por cuanto arde con gran facilidad, a pesar de que raras veces se quema su roseta completa. En todo caso se ha descartado tal carácter, aunque se le emplea como combustible.

Todas estas comunidades arbustivas son altamente inflamables en las temporadas de fuegos. Especialmente porque muchas de ellas son deciduas de verano y por su abundante estrato herbáceo, que suele acompañarlas, particularmente cuando el invierno ha tenido precipitaciones importantes. Por otra parte es reconocido que debido a la intervención humana, en el piemont y montañas costeras de la zona mediterránea predominan actualmente agrupaciones secundarias como resultantes de las intensas degradaciones a que fue expuesto el bosque primitivo. Entre ellas, el rol de los fuegos ha sido uno de los grandes causantes del desarrollo posterior de una formación que hoy día es denominada "matorral esclerófilo".

Otras comunidades parecen ser el producto final de una serie de alteraciones de bosques esclerófilos o primitivos matorrales, como por ejemplo la expansión del "espinal" de Acacia caven y al matorral bajo de Baccharis rosmarinifolia. Al desarrollar una fisonomía abierta, estas comunidades están expuestas a gran radiación solar y su abundante estrato herbáceo en gran parte se deshidrata intensamente durante el seco verano, lo cual favorece su combustibilidad. Estas agrupaciones cubren actualmente superficies importantes de la zona central, aproximadamente entre $\operatorname{los} 33^{\circ}$ y $37^{\circ} \mathrm{S}$.

La formación matorral actualmente es una agrupación expandida en todo el centro de la zona mediterránea del país, dominada por arbustos esclerófilos y árboles inmaduros. A menudo puede mezclarse con vegetación abierta tipo parque, vegetación graminoide herbácea o brezales. Según las condiciones de humedad e intervención antrópica, puede presentarse como una agrupación densa y a veces abierta, en que a la proximidad de las copas de los arbustos vecinos se suma el estrato herbáceo, el cual se seca a fines de primavera para conformar así una situación que favorece el rápido avance del fuego en verano.

Otro factor de incendio en las plantas del matorral, es la mantención de la actvidad de las hojas por períodos de uno o dos años, excepcionalmente tres o cuatro, lo que permite 
la existencia de un permanente estrato de hojas caídas con diferentes grados de descomposición. A esto se suma la existencia de plantas que botan ramas completas, trozos de corteza y otros elementos cuyo conjunto constituye un excelente material susceptible de ser quemado. Estos factores se ven incrementados por la presencia de otras características que hacen muy combustible la vegetación. Por ejemplo la existencia de resinas, aceites esenciales, pelos, espinas, un bajo contenido de agua y las gruesas cutículas presentes en las hojas de las plantas dominantes favorecen la combustión (AVILA et al. 1988).

Considerando gran parte de estos elementos y siguiendo la metodología de TRABAUD (1981) se estableció una carta de combustibilidad de las formaciones vegetales, para procurar determinar con mayor grado de precisión el grado de inflamabilidad de las agrupaciones presentes (QUINTANILLA 1998 y Fig.6).

También en la combustibilidad de la vegetación influye la calidad de la madera, a menudo rica en sustancias inflamables y de bajo contenido en minerales y esto está muy ligado a los intereses del hombre, en cuanto a seleccionar especies para leña y carbón. Precisamente esta actividad antrópica en el monte, es una de las principales responsables de fuegos en los cerros de las cadenas costeras. Indudablemente, al quemarse reiteradas veces el tapiz vegetal, se va generando un debilitamiento en la estructura y fisonomía de las comunidades afectadas particularmente en las laderas de solana, donde la vegetación es menos densa y se encuentra más deshidratada. Por lo mismo, la regeneración es más lenta que en los individuos de laderas en umbría. Con posterioridad al incendio, la reaparición de arbustos de rápido crecimiento es notoria en laderas más abiertas (Satureja gilliesi, Flourensia thuriphera, Lithrea caustica), notoriamente en el colihue (Chusquea cumingii) expuesto a la umbría (Fig.7).

Entre estos primeros resultados obtenidos, se comprueba la gran similitud del efecto del fuego en los estratos arbustivo y arbóreo, si se compara con otros casos estudiados (VILLASEÑOR y SAIZ 1990, QUINTANILLA 1996).

\section{CONCLUSIONES}

Se observa un enrarecimiento de las comunidades en situaciones post-incendio al introducirse plantas pioneras de rápido crecimiento - arbustivas y herbáceas - que van reemplazando paulatinamente la primitiva densidad y cobertura de estas agrupaciones, acarreando a menudo un empobrecimiento florístico de ellas.

Aunque hay matorrales arbóreos resistentes al fuego o de buena regeneración, se observa una degradación de los ecosistemas. Se constata que numerosos arbustos han pasado a la categoría de invasores Esto va modificando la estructura del matorral arbóreo.

\section{AGRADECIMIENTOS}

Este estudio fue realizado con el apoyo del Proyecto FONDECYT No 1990894 y del Departamento de Investigaciones (DICYT) de la Universidad de Santiago de Chile.

\section{REFERENCIAS}

ARAYA S. y G. AVILA, 1981. Rebrote de arbustos afectados por el fuego en el matorral chileno. An. Museo Hist. Natural. Valparaíso, 14: $107-113$.

AVILA G., M. ALJARO y G. MONTENEGRO , 1988. Incendios en la vegetación mediterránea de Chile. In: Fuentes E. y S. Prenafreta (Eds.), Ecología del paisaje de Chile Central. Ediciones Universidad Católica de Chile : 81 -86.

BALDUZZI A., I. SEREY ,,R. TOMASELLI y R. VILLASEÑOR, $1980=1981$. New phytosociological observations on the Mediterranean type of climax vegetation of central Chile. ATTI. Ins. Bot. Lab. Critt. Universitá de Pavia s.6 XIV 93-112.

BRAUN-BLANQUET, J., 1951. Planzensoziologie, Grundzüge der Vegetationskunde. 2é edit. Wien, Springer. CHUVIECO E., F. SALAS y J.M. MARTÍNEZ, 1997-1998.Geografía e incendios forestales. Serie Geográfica 7. Universidad de 


\section{Alcalá Henares : 11-18}

DONOSO, C., 1990. Ecología Forestal. El bosque y su medio ambiente. Ed. Universidad Austral de Chile, Valdivia.

JULIO, G., 1995. Actas del Taller Internacional Prognosis y Gestión en control de incendios forestales: Actas del Taller Internacional. Santiago de Chile. Proyecto FONDEF FL-13, CONICYT- Universidad de Chile. Instituto Forestal-CORFOINTEC.Santiago.

\section{MILLAN M., M. STRELA y C. BADENAS} 1998. Meteorological Processes Relevant Forest Fire Dynamics on the Spanish Mediterranean Coast. American Meteorological Society : $83-100$

PAPIÓ C. y L. TRABAUD, 1999. Comparative study of the Aerial Structure of Five Shrubs of Mediterranean Schrublands. Forest Science, 31 (1) : 146-159.

QUINTANILLA， V. 1975. La carta bioclimática de Chile central. Revista Geográfica de Valparaíso, $6: 33$ - 58.

QUINTANILLA, V. 1996 Alteraciones por el fuego en la Cordillera de la Costa de Chile mediterráneo. Antecedentes en un parque nacional. Revista Pireneos, 142 : 119-134.

QUINTANILLA, V. 1983. Biogeografía de Chile. Vol. III de Geografía de Chile. Ed. Instituto Geográfico Militar. Santiago.
QUINTANILLA, V. 1998a. Geocartography and fires of vegetation in central Chile. In: Ann. III International Conference on Forest Fire Research. University of Coimbra. Portugal : 10221030.

QUINTANILLA, V. 1998b. Los incendios de vegetación en el cordón costero de Chile central. El apoyo de la cartografía para su gestión en la prevención y análisis. Caso de estudio. Contrib. Cient. y Tec. Area Ingeniería, 120. Universidad de Santiago de Chile. Santiago.

QUINTANILLA, V.1999. Géocartographie et feu de végétation au Chili Tempéré. In : to 19th. International Cartographic Conference. Otawa. $348-360$.

TRABAUD, L.1981. Man and fires impact of mediterranean vegetation. In: di Castri , Goodall y Zeprech (Eds.), Mediterranean types schrubslands Ecosystems. Elsevier Scientific Pub. : 523 - 537.

VERA, V. y V. QUINTANILLA , 1997. Incendios forestales en la V Región: Características y distribución espacial tras diez años de ocurrencia. Anales Soc. Chilena de Cs. Geográficas: 471 - 479.

VILLASEÑOR, R. y F. SAIZ , 1993. Incendios forestales en el Parque Nacional La Campana, sector Ocoa, Vta. Región de Chile. Efectos sobre el estrato arbustivo arbóreo. An. Mus. Hist. Natural, Valparaíso, 21: 15 - 26. 


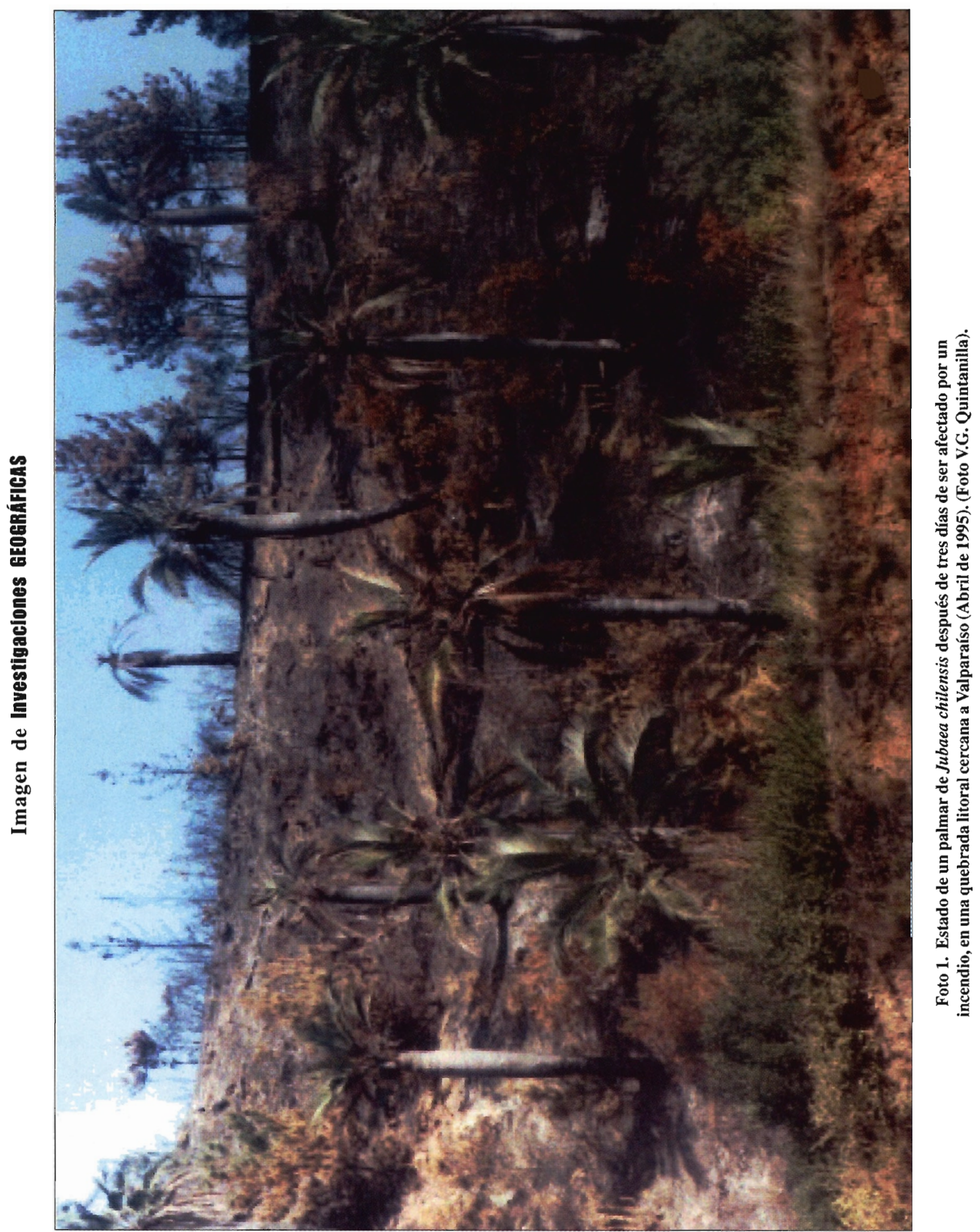

